\title{
Quick Observation of Tissues in Solution by Atmospheric Scanning Electron Microscopy (ASEM)
}

Nassirhadjy Memtily, 1, 2,3 Mari Sato ${ }^{2}$, Tomoko Okada ${ }^{2}$, Tatsuhiko Ebihara ${ }^{2}$, Kazuhiro Mio ${ }^{1,2}$, Mitsuo Suga ${ }^{4}$, Hiditoshi Nishiyama ${ }^{4}$, Chikara Sato ${ }^{1,2}$

1. Graduate School of Comprehensive Human Sciences, University of Tsukuba. 1-1-1 Tennodai, Tsukuba, Ibaraki Prefecture 305-0006, Japan.

2. Biomedical Research Institute, National Institute of Advanced Industrial Science and Technology

(AIST), Higashi 1-1-1, Tsukuba, Ibaraki 305-8566, Japan.

3. Traditional Uyghur Medicine Institute of Xinjiang Medical University, 393 Xinyi Rd, Urumqi, Xinjiang Uyghur Autonomous Region, 830011 China.

4. Advanced Technology Division, JEOL Ltd., Musashino 3-1-2, Akishima, Tokyo 196-8558, Japan.

Correspondence should be addressed to Chikara Sato (ti-sato@aist.go.jp).

The structure-function relationships of tissues are key to understand their mechanisms, which should be precisely studied. Optical microscopy $(\mathrm{OM})$ has been developed in various ways and has led to great discoveries and advances in biology and medicine [1,2]. The tissue samples are prepared by paraffin embedding or quickly frozen. Cryo-thin-sectioning is used for intra-operative cancer diagnosis mainly based on cellular nucleus size, although it is difficult and the whole procedure takes about 15-30 minutes for each sample [3]. Standard electron microscopy (EM) has subnanometer or nanometer resolution, but samples must be observed in vacuum. For biological samples, transmission electron microscopy (TEM) observation, requires time-consuming tissue preparation including fixation, dehydration, embedding, ultra-thin sectioning and staining. On the other hand, in the atmospheric scanning electron microscope (ASEM), a $2-3 \mu \mathrm{m}$-thick layer of the sample can be observed at high resolution without dehydration [3-5]. The applicability of ASEM for the quick observation of cancer-metastasized tissue and normal tissue in liquid was studied.

ASEM was successfully used for the observation of cerebellum at high resolution, and a quick staining method for intra-operative cancer diagnosis was further developed. Excised mouse tissue slabs (cerebrum, cerebellum, spinal cord, optic nerve fiber, kidney, liver, esophagus, stomach, intestine, cardiac, tongue, ear skin and skeletal muscle) were fixed with $4 \%$ paraformaldehyde (PFA) and $1 \%$ glutaraldehyde (GA), perforated with Triton X-100, stained with phosphotungstic acid (PTA) for 3 hrs, and imaged immersed in $10 \mathrm{mg} / \mathrm{ml}$ glucose solution by ASEM at $30 \mathrm{kV}$ acceleration voltage [6]. In cerebellum, three distinct cortical layers were observed (the molecular layer, the Purkinji cell layer and the granular layer) as well as cerebellar white matter (Fig. 1B). Neurons were connected by delicate systematic networks (Fig. 1C and D). At a higher magnification, nuclei including brightly stained patches, presumably heterochromatin and nucleoli were observed (Fig. 1D). These results are consistent with those obtained by hematoxylin-eosin (HE) staining and OM (Fig. 1A).

Control lung tissue and lung tissue metastasized by breast cancer were also fixed, quickly stained with uranyl acetate (UA) for just 15 minutes without Triton X-100 treatment, and observed by ASEM. The nuclei located close to the surface of tissue slabs were clearly observed, and the size difference between normal and cancer cells was clear (Fig. 2). These results suggest the potential of the quick method for intraoperative cancer diagnosis. The results were comparable with those obtained when the tissue slabs were treated with Triton X-100 and stained with TI-Blue and PTA (data not shown). 


\section{References}

[1] C Golgi, Bollettino dellaSocietà Medico-Chirurgica di Pavia, 13 (1898), p. 1-14.

[2] SW Hell, Science, 316 (2007), p.1153-1158.

[3] C Sato, JEOL news, 43 (2011), p.13-20.

[4] H Nishiyama et al, J Struct Biol, 172 (2010), p. 191-202.

[5] Y Maruyama et al, J Struct Biol, 180 (2012), p. 259-270.

[6] N Memtily et al, International J Oncology, in press.
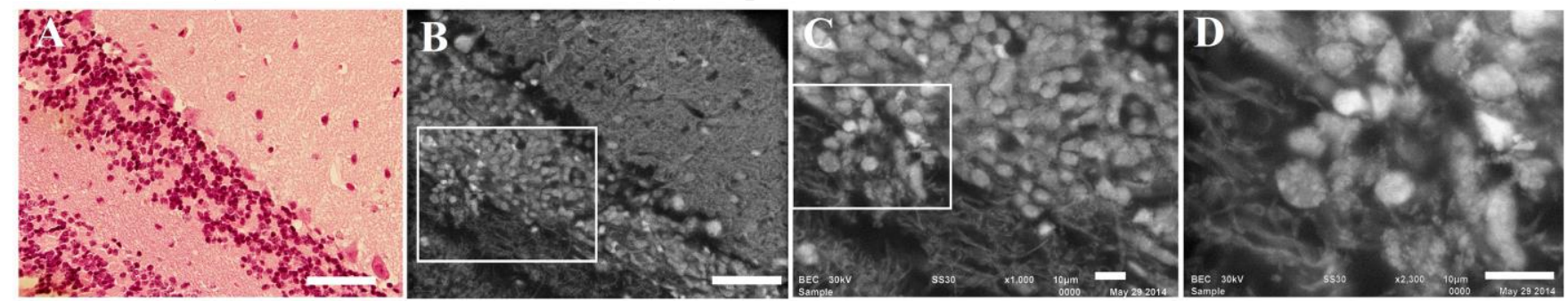

Figure 1. (A) OM of an HE-stained horizontal thin-section of cerebellum, and ASEM images of a PTA-stained cerebellum slab (B-D). (B) Three clearly different layers - the molecular layer, Purkinji cell layer, the granular layer - are visible as well as cerebellar white matter. (C, D) Higher magnification image of the white rectangle in the preceeding panel. Scale bar $50 \mu \mathrm{m}$ in A and B panel, $10 \mu \mathrm{m}$ in C, D.

Normal lung
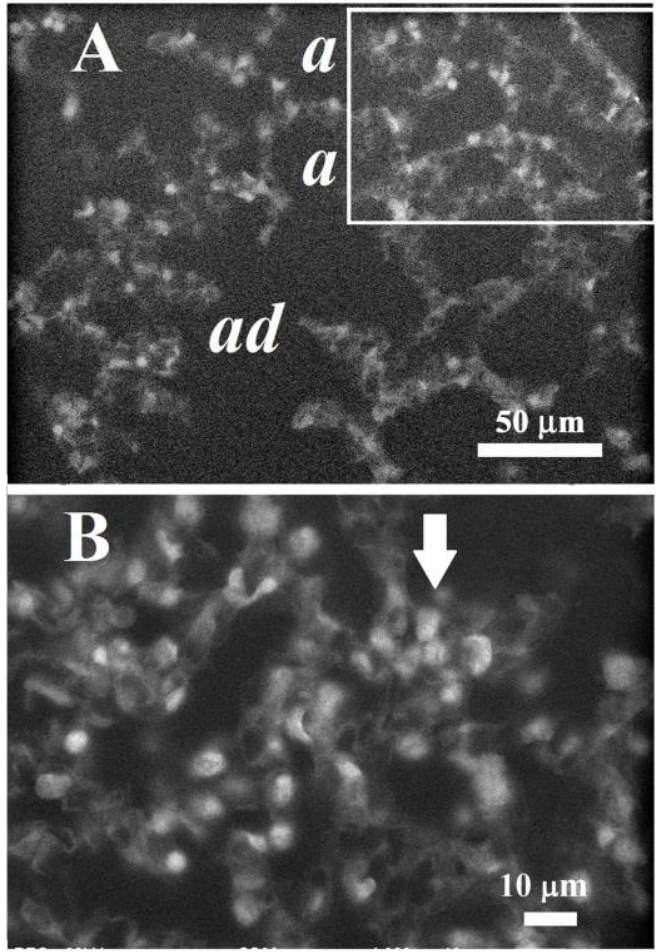

Cancer metastasized lung
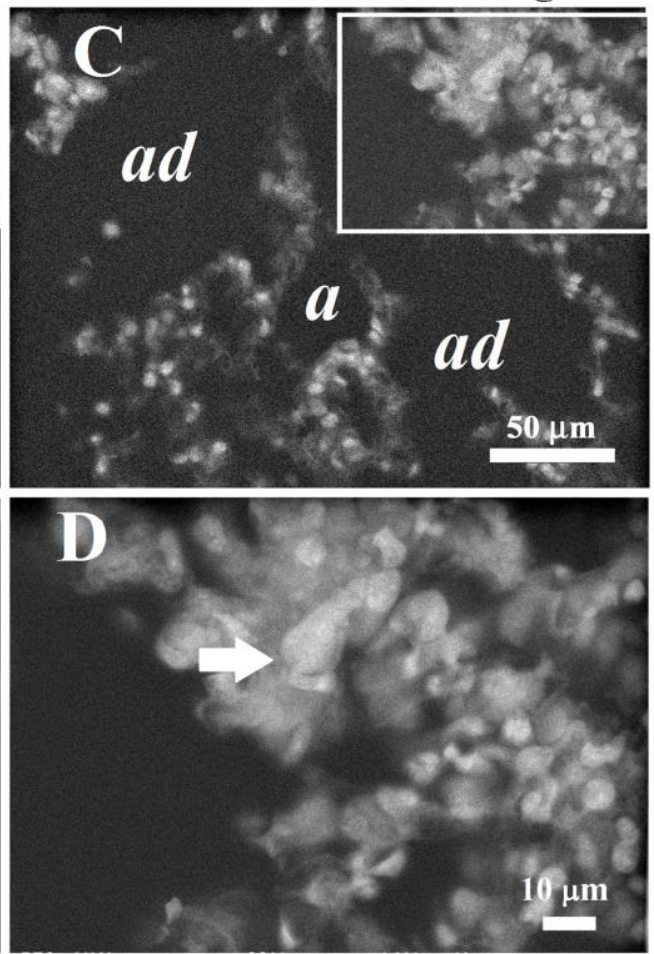

Figure 2. Quick observation of lung tissue (A, B) and lung metastasized by breast cancer cells (C, D) by ASEM. Fixed lung tissue slabs without perforation were quickly stained with UA for 15 minutes, and observed by ASEM. (A, B) Normal lung. A normal thin wall structure with alveoli $(a)$ and alveolar ducts $(a d)$ was observed. The cell nuclei (arrow) located at the surface of the slab were brightly imaged. (C, D) Lung metastasized by breast cancer cells. Lung structures look fainter in the metastasized area (top right). Instead, the region was occupied by cells with unusually large nuclei (arrow). Scale bar: 50 $\mu \mathrm{m}$ in $\mathrm{A}, \mathrm{C} ; 10 \mu \mathrm{m}$ in $\mathrm{B}, \mathrm{D}$. 